\title{
Possible role of rf melted microparticles on the operation of high-gradient accelerating structures
}

\author{
G. S. Nusinovich, D. Kashyn, and T. M. Antonsen, Jr. \\ Institute for Research in Electronics and Applied Physics, University of Maryland, College Park, Maryland 20742-3511, USA
}

(Received 14 May 2009; published 13 October 2009)

\begin{abstract}
High-gradient accelerating structures should operate reliably for a long time. Therefore studies of various processes which may lead to disruption of such an operation are so important. In the present paper, the dissipation of rf electromagnetic energy in metallic microparticles is analyzed accounting for the temperature dependence of the skin depth. Such particles may appear in structures, for example, due to mechanical fracture of irises in strong rf electric fields. It is shown that such microparticles with dimensions on the order of the skin depth, being immersed in the region of strong rf magnetic field, can absorb enough energy in long-pulse operation to be melted. Then, the melted clumps can impinge on the surface of a structure and create nonuniformities leading to field enhancement and corresponding emission of dark current. Results are given for several geometries and materials of microparticles.
\end{abstract}

DOI: 10.1103/PhysRevSTAB.12.101001

PACS numbers: 29.20.Ej, 29.20.D-

\section{INTRODUCTION}

In spite of the long history of the development of highgradient accelerators, there are still many explanations for rf breakdown at high gradients. These explanations and their corresponding models were recently overviewed in Ref. [1], which includes references to the original papers by Wilson [2] and others who proposed these models. For example, it was suggested in Ref. [3] and repeated elsewhere [1] that a small piece of metal can be moved away from a protrusion located in the region of a strong $\mathrm{rf}$ electric field and can be bombarded by field emitted electrons to such a degree that it is ionized, forms a local plasma, and finally causes rf breakdown. The nature of field emission from microparticles was thoroughly analyzed in Ref. [4].

In what follows we focus on another role the microparticles can play in the operation of high-gradient accelerating structures. We show that once a microparticle is extracted from a protrusion and moves from the region of strong $\mathrm{rf}$ electric field to a region of strong rf magnetic field, it can be melted without any bombardment. Then such a melted particle or clump can impinge on the surface of a structure forming local nonuniformity, which may initiate further events.

\section{DISSIPATION OF ELECTROMAGNETIC ENERGY IN SMALL MICROPARTICLES}

Scattering and dissipation of electromagnetic waves by small microparticles having dimensions much smaller than a wavelength was analyzed by Landau and Lifshitz [5] with a reference to the original treatise by Rayleigh [6] who studied the scattering, but not dissipation of light by small particles. At present, the losses in small particulates irradiated by electromagnetic waves are studied in various areas of physics research; see, e.g., Ref. [7] and references therein. As follows from Sec. 73 in Ref. [5], the rf energy dissipated in a small microparticle during a pulse of duration $\tau$ is equal to

$$
W=\frac{\omega \tau}{2} V\left(\alpha_{e}^{\prime \prime} \varepsilon_{0}|\vec{E}|^{2}+\alpha_{m}^{\prime \prime} \frac{1}{\mu_{0}}|\vec{H}|^{2}\right) .
$$

Here $\omega$ and $\vec{E}(\vec{H})$ are the frequency and electric (magnetic) field amplitude of the wave, $\tau$ is the pulse duration of the rf field, $V$ is the volume of microparticle, and $\alpha_{e}^{\prime \prime}$ and $\alpha_{m}^{\prime \prime}$ are imaginary parts of the electric and magnetic polarizabilities of the microparticle.

Without going into detail of the derivation of formulas for the polarizabilities described in the textbook [5] let us briefly explain the method. The wave fields [electric (for $\alpha_{e}$ ) and magnetic (for $\alpha_{m}$ )] are written for the spaces inside and outside the particle accounting for the particle geometry; the field in the external region, obviously, contains two components - the external quasiuniform field and the perturbation caused by the microparticle. Then, at the particle surface the relevant components of these fields are matched separately according to the known boundary conditions.

The simplest configuration of a microparticle that can be used for initial estimates is a sphere. For a small sphere of radius $a$ the volume in (1) is $V=(4 \pi / 3) a^{3}$ and for metals the imaginary part of the electric polarizability of such a microparticle, in accordance with (72.2) of Ref. [5], is given by

$$
\alpha_{e}^{\prime \prime}=\frac{9}{4 \pi} \frac{\varepsilon^{\prime \prime}}{4+\left(\varepsilon^{\prime \prime}\right)^{2}},
$$

where $\varepsilon^{\prime \prime}=4 \pi \sigma / \omega(\sigma$ is the conductivity of the given metal). Correspondingly, the imaginary part of the magnetic polarizability is given in the solution of problem 1 in Sec. 45 of Ref. [5] as

$$
\alpha_{m}^{\prime \prime}=-\frac{9}{16 \pi}\left(\frac{\delta}{a}\right)^{2}\left[1-\frac{a}{\delta} \frac{\sinh (2 a / \delta)+\sin (2 a / \delta)}{\cosh (2 a / \delta)-\cos (2 a / \delta)}\right] .
$$




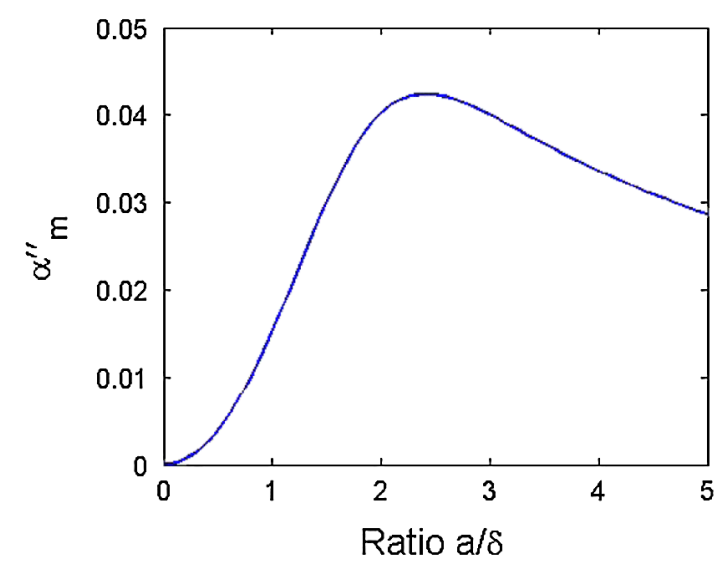

FIG. 1. (Color) Imaginary part of the magnetic polarizability of a small sphere as a function of the ratio of the sphere radius to the skin depth.

This polarizability depends only on the ratio of the sphere radius to the skin depth $\delta(m)=1 / \sqrt{\pi f \mu \sigma}$. This dependence is shown in Fig. 1 from which it follows that its maximum corresponds to the sphere radius of about 2.4 skin depths. For example, the skin depth in the case of a copper particle irradiated by the X-band wave (let us take the frequency of SLAC structures developed for the next linear collider, $f=11.424 \mathrm{GHz}$ ) is about $0.6 \mu \mathrm{m}$. So the maximum absorption of the microwave power will be in the case of microparticles with a diameter of a few microns. Note that the dependences shown in Fig. 1 and also in Fig. 2 are quite similar to those given in Figs. 2 and 3 of Ref. [7].

As follows from comparison of (2) with (3) and Fig. 1, the magnetic polarizability plays the dominant role in determining the microwave energy dissipated in a metallic sphere of a radius on the order of a skin depth. The contribution from the electric field becomes important only when the radius of the microparticle is much smaller than $1 \mathrm{~nm}$ independent on the operating wavelength. (This

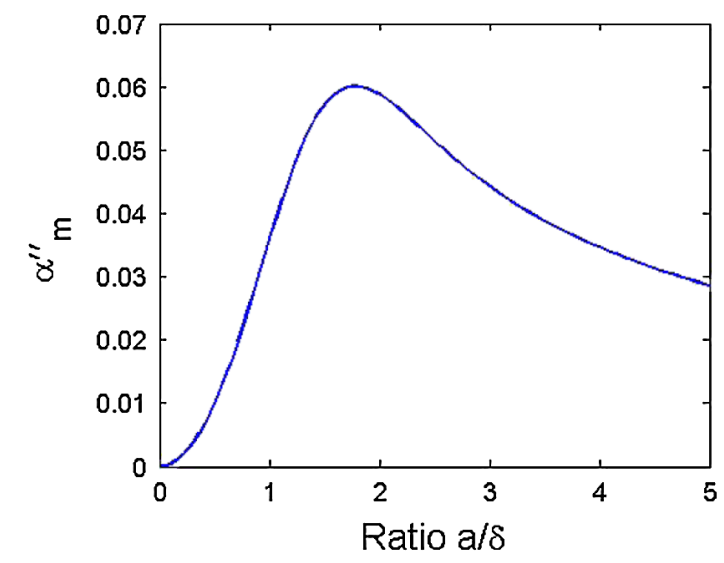

FIG. 2. (Color) Imaginary part of the magnetic polarizability of a small cylinder oriented perpendicularly to the direction of the magnetic field. conclusion agrees with the one made in Ref. [7] where it was found that the effect of the electric field becomes important only when the radius of a small sphere is much smaller than the skin depth.) Strictly speaking, when such a small metallic sphere is located close to the surface of the accelerating structure, there can be significant enhancement of the electric field: this issue is discussed in Ref. [8] with a reference to Rohrbach [9]. In what follows, however, we will not consider this case and therefore will neglect $\alpha_{e}^{\prime \prime}$ in our analysis.

In the same fashion, one can evaluate the rf energy dissipation in microparticles of a different shape. For instance, Landau and Lifshitz [5] in the second and third problems of Sec. 45 consider a small metallic cylinder of a radius $a$ oriented perpendicular and parallel to the direction of the magnetic field of the wave. For the magnetic polarizability in the case of a perpendicularly oriented cylinder they give the following formula:

$$
\alpha_{m}=-\frac{1}{2 \pi}\left[1-\frac{2}{k a} \frac{J_{1}(k a)}{J_{0}(k a)}\right] .
$$

In (4) $k=(1+i) / \delta$ is the complex wave number, hence the Bessel functions depend on a complex argument. In such a case, as known in the literature describing the processes in imperfect conductors [10], these functions should be expressed via Kelvin functions ber $_{0,1}(x)$ and $\operatorname{bei}_{0,1}(x)$ [11]. The dependence of the imaginary part of the magnetic polarizability (4) on the ratio of the cylinder radius to the skin depth obtained with the use of these functions is shown in Fig. 2 which looks quite similar to Fig. 1. Now, the peak value of this polarizability is higher ( 0.06 versus 0.042 for a sphere) and the corresponding ratio of the radius to the skin depth yielding the maximum polarizability is smaller (1.8 versus 2.4 for a sphere). When a cylinder is oriented parallel to the direction of the magnetic field its polarizability is 2 times smaller [5]. Note that in Ref. [5] only the limiting cases of small and large radius in comparison with the skin depth are considered. As follows from Ref. [5], the imaginary part of magnetic polarizability of a cylinder perpendicular to the wave magnetic field in the case of a radius much smaller than the skin depth is 2.5 times larger than that for a sphere, while in the opposite case of a large radius it is almost the same (corresponding coefficient is $1 / 2$ for a cylinder versus $9 / 16$ for a sphere). In the latter case (when a microparticle can be treated as a macroparticle), Eqs. (1)-(4) yield for the density of losses the same dependence (proportionality to $\omega \delta$ ) as the usual rf surface loss formula.

\section{CRITICAL FIELDS AND PULSE DURATION}

The dissipation of microwave energy defined by (1) leads to a temperature rise for this microparticle. Then, after the temperature reaches the melting point some energy should also be spent to melt the microparticle. Correspondingly, the energy balance describing the pro- 
cess of heating and melting a microparticle can be represented as

$$
W=W_{H}+W_{M},
$$

where the first and second terms in the right-hand side correspond to the energy required for heating up to the melting temperature and for melting, respectively. In the same fashion, all the process can be subdivided into two stages.

First stage: temperature rise to the melting point.During the first stage $\left(0 \leq t \leq t_{H}\right)$, the temperature increases up to the melting temperature. In this stage, the energy balance equation, in line with (1) and the standard definition of the temperature rise, has the form

$$
\frac{\omega t}{2} \alpha_{m}^{\prime \prime} \frac{1}{\mu_{0}}|H|^{2}=c_{T} \rho_{d} \Delta T .
$$

In (6), $c_{T}$ is the specific heat capacity and $\rho_{d}$ is the density of a given metal. When the temperature rises up to the melting point ( $\Delta T=T_{m}-T_{0}$ ), Eq. (6) determines the corresponding instant of time $t=t_{H}$.

Let us emphasize that Eq. (6) contains the imaginary part of the magnetic polarizability which depends on the ratio $a / \delta$, which, in turn, can be temperature dependent. In fact, the temperature expansion of the radius $a$ with the temperature rise can be neglected because for typical materials (such as copper, aluminum, tungsten, or molybdenum) it is smaller than $2 \%$. However, the changes in the skin depth can be significant. As follows from the BlochGruneisen law which predicts a linear dependence of the material resistivity on temperature for temperatures exceeding $2 / 3$ of the Debye temperature (which is valid for temperatures equal to and exceeding the room temperature), the skin depth is proportional to $\sqrt{T}$. Correspondingly, the argument for the functions defining the magnetic polarizability (3) and (4) can be redefined as

$$
\frac{a}{\delta}=\left(\frac{a}{\delta}\right)_{0} \sqrt{\frac{T_{0}}{T}}
$$

Accounting for the nonlinear dependence of $\alpha_{m}^{\prime \prime}$ on temperature allows us to rewrite (6) in dimensionless variables as an ordinary first-order differential equation

$$
\frac{d y}{d t^{\prime}}=\Psi(z)
$$

In (8), $y=\Delta T / T_{0}$ is the temperature rise normalized to the initial temperature, $t^{\prime}=(\omega t)|H|^{2} / 2 T_{0} \rho_{d} c_{T}$ is a normalized time, the function $\Psi(z)$ describes the dependence of the imaginary part of the magnetic polarizability defined for specific geometries by (3) and (4), and its argument denotes the radius-to-the skin depth ratio (7), i.e. $z=$ $(a / \delta)_{0} / \sqrt{1+y}$.

The solution of (8) is shown in Fig. 3 for two values of the initial ratio $(a / \delta)_{0}-2.0$ and 4.0 by solid lines. Dashed lines show asymptotic solutions obtained for the case when

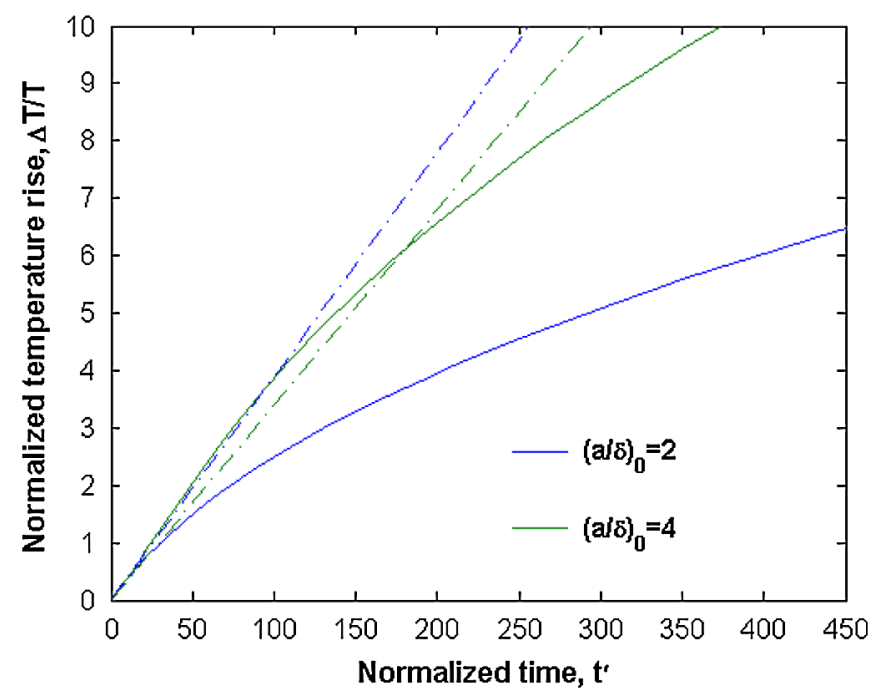

FIG. 3. (Color) Temperature rise (normalized to the initial temperature) as a function of normalized time for a microsphere with the temperature dependence of the skin depth taken into account (solid lines) and ignored (dashed lines).

the temperature dependence of conductivity in the skin depth is ignored. While the asymptotic solutions shown by straight dashed lines have obvious interpretation, the curves shown by solid lines exhibit more interesting behavior. Initially, when the temperature rise is small, the temperature rise for the case $(a / \delta)_{0}=2.0$ exceeds the temperature rise in the case $(a / \delta)_{0}=4.0$. However, as the temperature rise becomes larger, the dissipation in the case $(a / \delta)_{0}=2.0$, in line with data shown in Fig. 1, rapidly decreases, while the dissipation in the case $(a / \delta)_{0}=4.0$ increases because the ratio $a / \delta$ decreases with the temperature rise. They become equal when the temperature rise is about 0.3 of the initial temperature. Then, as is seen in Fig. 3, the dissipation and corresponding temperature rise in the case $(a / \delta)_{0}=4.0$ becomes much larger than in the case $(a / \delta)_{0}=2.0$. Comparison of the temperature rise in a microsphere with an optimal initial ratio $(a / \delta)_{0}=2.3$ with the temperature rise in a microsphere having initial ratio $(a / \delta)_{0}=5.0$ shows that the temperatures become equal much later, when the temperature rise is about 1.6 of the initial temperature.

To correctly interpret the data shown in Fig. 3, one should estimate the melting-to-initial temperature ratio for specific materials. For example, for such materials as copper, aluminum, tungsten, and molybdenum, the $(a / \delta)$ ratio decreases at the melting point by $2.1,1.76,3.5$, and 3.26 times with respect to initial values, respectively. This means, first, that one should take this effect into account when the optimal ratio $(a / \delta)$ is estimated based on the data shown in Figs. 1 and 2. Now, these optimal values (2.4 for the sphere and 1.8 for the cylinder) should correspond to melting temperatures. Correspondingly, optimal initial values of these ratios are $\sqrt{T_{m} / T_{0}}$ times larger. (Here the word 
"optimal" means that particles of this size will be melted faster than others.) For example, in the case of a copper microsphere, the optimal value of the initial ratio $(a / \delta)_{0}$ is about 2.1 times larger than 2.4, i.e., the optimal initial radius is close to 5 times initial skin depth. Then, the curves like those shown in Fig. 3 should be used for estimating the instant of normalized time corresponding to the temperature rise from the initial to the melting temperature, viz. for example, for copper $y_{\max }=\left(T_{m}-T_{0}\right) / T_{0} \approx 3.5$. As follows from Fig. 3, in this case for a copper microparticle with $(a / \delta)_{0}=4.0$ the normalized time required for reaching the melting temperature is about 80 . By using normalization of this time variable given after (8), one can easily convert this value into the required time interval for given amplitude of the rf magnetic field and other parameters.

Second stage: melting.-During the second stage $\left(t_{H} \leq\right.$ $t \leq \tau)$, the energy balance equation can be written as follows:

$$
\frac{\omega\left(\tau-t_{H}\right)}{2} \alpha_{m}^{\prime \prime} \frac{|H|^{2}}{\mu_{0}}=\rho_{d} \Lambda
$$

where $\Lambda$ is the specific melting heat. This equation determines the time interval required for melting a heated microparticle in a magnetic field of given amplitude.

The dependence of the critical magnetic field defined as the value of magnetic field that, first, causes the temperature to rise to the melting point and, then, melt a particle is shown for several metals in Fig. 4 for spherical microparticles with different initial values of the $(a / \delta)_{0}$ ratio. Asymptotic solutions obtained ignoring the temperature dependence of the skin depth are shown by dash-dotted lines. As one can find, the discrepancy between asymptotic solutions and exact ones is larger when the initial ratio $(a / \delta)_{0}$ equals 2 [Fig. 4(a)] because in this case the tem- perature rise causes a large decrease in the imaginary value of the magnetic polarizability shown in Fig. 1. As follows from these results, in strong enough fields of long pulse duration such microparticles can be melted. Consideration of a cylinder oriented perpendicularly to the wave magnetic field yields similar results. Results shown in Fig. 4 were obtained neglecting the radiation losses. To prove this assumption let us note that, for example, the energy required for melting a copper spherical microparticle with the radius of $1.3 \mu \mathrm{m}$ is about $4.8 \times 10^{-8}$ Joules, while, as follows from the Stefan-Boltzmann law, the power of blackbody radiation from such a sphere at the melting temperature is close to $3.7 \times 10^{-6} \mathrm{~W}$ and, hence, in microsecond pulses the radiated energy is equal to $3.7 \times$ $10^{-12}$ Joules, i.e., about 4 orders of magnitude smaller than what is needed to melt the particle. There is also another possible mechanism for the heat sink from microparticles, viz. the heat transport to the bulk of the structure in the case of their contact. This issue was briefly discussed in Ref. [4]. Importance of this mechanism strongly depends on the geometry of a microparticle: for an ideal solid sphere the effect is negligibly small, while, for example, for a small cylinder standing on the structure surface it can be significant. Therefore, it is impossible to draw here any general conclusion about it.

\section{DISCUSSION AND SUMMARY}

The results of our simple considerations show that it is quite possible that microparticles of a micron size can be melted in strong rf magnetic fields of long enough pulse duration. Magnetic fields and pulse durations required for melting shown in Fig. 4 are close to those used in SLAC experiments with $\mathrm{X}$-band standing-wave structures $[12,13]$. For example, results reported in Ref. [13] indicate
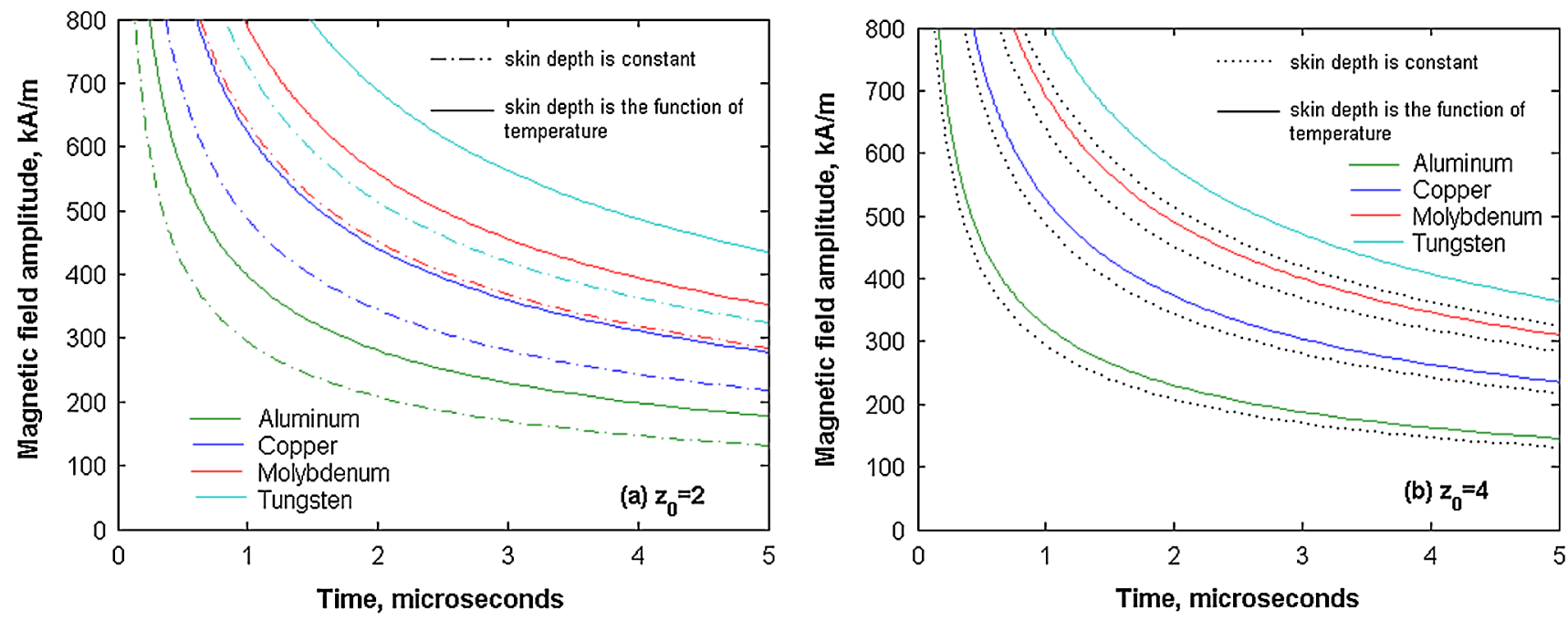

FIG. 4. (Color) Critical field amplitude leading to melting of spherical microparticles with the initial $(a / \delta)$ ratio equal to $2.0(a)$ and 4.0 (b). The $\mathrm{rf}$ frequency is equal to $11.4 \mathrm{GHz}$. 


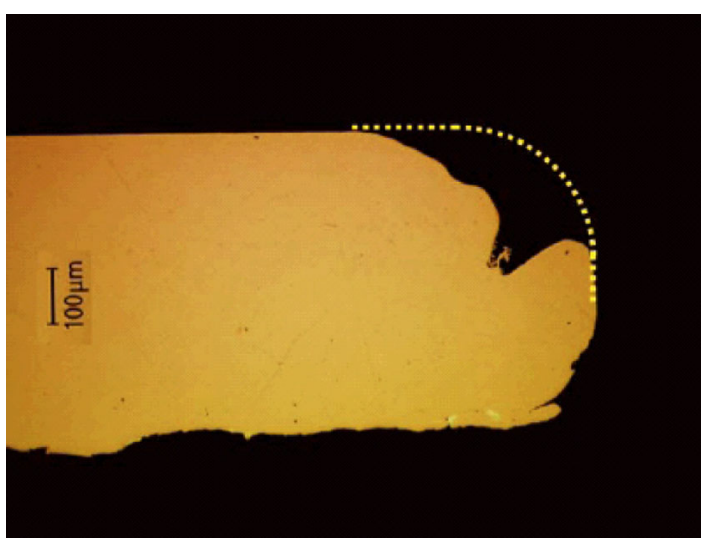

FIG. 5. (Color) Input coupler iris (the dotted line represents the iris geometry before $\mathrm{rf}$ processing); this figure is reproduced from Ref. [15].

that in standing-wave $\mathrm{X}$-band structures the accelerating gradient of $70 \mathrm{MV} / \mathrm{m}$ results in the surface magnetic field at the inner edge of the iris of about $700 \mathrm{kA} / \mathrm{m}$ and the pulse duration in those studies is in the range of $400 \mathrm{~ns}$. This field is in the range of magnetic fields shown in Fig. 4: for such materials as aluminum the critical field at $400 \mathrm{~ns}$ is lower than $700 \mathrm{kA} / \mathrm{m}$, while for others this field becomes critical at pulse durations close to $1 \mu \mathrm{m}$.

Let us now briefly discuss the possible origin of such microparticles and the consequences of their melting on the operation of high-gradient structures. It seems possible that, in line with the "mechanical fracture" [3] and atomistic [14] models, microparticles may appear on the surfaces of input irises where the rf electric field is very strong because the outward electrostatic tension may cause the tip of an asperity on the iris surface to break off. The erosion of an input iris coupler was discussed in Ref. [15]. However, the mechanism responsible for the erosion was not identified there. An image of this damaged iris is shown in Fig. 5. Unfortunately, from the photo shown in Fig. 5 it is impossible to determine the size of microparticles extracted in each event. To answer this question it would be

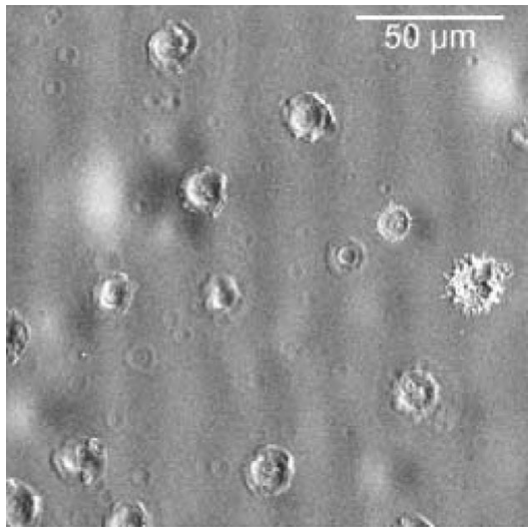

FIG. 6. Splashes on the metallic surface of a structure (reproduced from Ref. [16]). necessary to inspect structures after a short series of breakdown events. To the best of our knowledge, this was never done in high-gradient experiments. Therefore this photo serves here for illustrative purposes only.

Now let us briefly discuss the consequences of these events. Between rf pulses a melted clump of metal can hit a structure surface and cause the appearance of spots that are observed in numerous experiments. Examples of such surfaces are shown in Fig. 6. When a clump impinges the metallic surface and forms a spot with a radius of a few microns, as shown in Fig. 6, the height of the rims of the spot is less than a micron, which is enough for creating significant nonuniformity and, hence, magnification of the rf electric field resulting in the field emission of a dark current there. The radius of 5 skin depth in copper translates to about $3 \mu \mathrm{m}$ size for wave frequency of $11.4 \mathrm{GHz}$.

Results of our study show that microparticles of a micron size can be melted in strong rf magnetic fields of long enough pulse duration. Then, these melted clumps can impinge on the structure surface and initiate further breakdown events. This scenario can be treated as an alternative to building up a small protrusion from the surface initially melted by a strong rf magnetic field (the case considered in Ref. [2]).

\section{ACKNOWLEDGMENTS}

This work is supported by the Office of High Energy Physics of DoE. The authors are indebted to V. Dolgashev for the discussion stimulating their interest in this problem.

[1] R. B. Palmer, R. C. Fernow, J. C. Gallardo, D. Stratakis, and D. Li, Phys. Rev. ST Accel. Beams 12, 031002 (2009).

[2] P. Wilson, in Proceedings of the 12th AAC Workshop, edited by M. Conde and C. Eyberger, AIP Conf. Proc. No. 877 (AIP, Melville, New York, 2006), p. 27.

[3] Z. Insepov, J. H. Norem, and A. Hassanein, Phys. Rev. ST Accel. Beams 7, 122001 (2004).

[4] H. Padamsee and J. Knobloch, High Energy Density Microwaves, edited by R. M. Phillips, AIP Conf. Proc. No. 474 (AIP, Woodbury, New York, 1999), p. 212.

[5] L.D. Landau and E. M. Lifshitz, Electrodynamics of Continuous Media (Pergamon Press, Oxford, 1960).

[6] Scientific Papers by Lord Rayleigh (Dover, New York, 1964), Vol. 1, p. 104.

[7] W. Tang, H. Bosman, Y. Y. Lau, and R. M. Gilgenbach, J. Appl. Phys. 97, 114915 (2005).

[8] J. W. Wang and G. A. Loew, Report No. SLAC-PUB-7684, 1997.

[9] F. Rohrbach, Report No. CERN 71-28, 1971.

[10] S. Ramo and J. R. Whinnery, Fields and Waves in Modern Radio (John Wiley \& Sons, New York, 1960), 2nd ed., p. 243.

[11] M. Abramowitz and I. A. Stegun, Handbook of Mathematical Functions, National Bureau of Standards, 1964, Sec. 9.9 and Table 9.12. 
[12] V. A. Dolgashev and S. G. Tantawi, Proceedings of the 6th Workshop on High Energy Density and High Power RF, edited by S.H. Gold and G. S. Nusinovich, AIP Conf. Proc. No. 691 (AIP, Melville, New York, 2003), p. 151.

[13] V. A. Dolgashev, Report No. SLAC-PUB-10114, 2002.

[14] F. Djurabekova and K. Nordlund, CLIC Breakdown Workshop, 2008; http://indico.cern.ch/materialDisplay. py? contribId=7\&sessionId $=2 \&$ material $I d=$ slides $\&$ confId $=33140$.

[15] W. Wuensch, in Proceedings of the 8th European Particle Accelerator Conference, Paris, 2002 (EPS-IGA and CERN, Geneva, 2002), p. 134.

[16] S. E. Harvey et al., Report No. SLAC-PUB-10197, 2003. 